\title{
Click chemistry on diterpenes: anti-inflammatory activity of the acetylenic derivatives of levopimaric acid and products of their transformations
}

\author{
Sergey F. Vasilevsky, ${ }^{\text {ad* }}$ Denis S. Baranov, ${ }^{a}$ Anastasia I. Govdi, ${ }^{a}$ Irina V. Sorokina, \\ Tatyana G. Tolstikova, ${ }^{\mathrm{b}}$ Genrikh A. Tolstikov, ${ }^{\mathrm{b}}$ and Igor V. Alabugin ${ }^{\mathrm{c}^{*}}$ \\ ${ }^{a}$ V.V. Voevodsky Institute of Chemical Kinetics and Combustion, SB of the Russian Academy of \\ Sciences, 3 Institutskaya str., 630090 Novosibirsk, Russian Federation \\ ${ }^{b}$ N.N. Vorozhtsov Novosibirsk Institute of Organic Chemistry, SB of the Russian Academy of \\ Sciences, 9 prosp. Acad. Lavrent'eva, 630090 Novosibirsk, Russian Federation \\ ${ }^{c}$ Department of Chemistry and Biochemistry, Florida State University, \\ Tallahassee, Florida 32306 \\ ${ }^{d}$ Instititute Novosibirsk State University, 2 Pirogova Str., 630090, Novosibirsk, Russian \\ Federation \\ E-mail:vasilev@kinetics.nsc.ru, alabugin@.chem.fsu.edu
}

DOI: $\underline{\text { http://dx.doi.org/10.3998/ark.5550190.0015.500 }}$

\begin{abstract}
Anti-inflammatory activity of amides of levopimaric acid (LpA) with acetylenic moiety and its esters with 4-R-triazolic substitutions was investigated using histamine-induced mice paw edema model. The synthesized LpA derivatives possess anti-inflammatory activity comparable to the activity of indomethacin.

Keywords: Acetylenes, Levopimaric acid, 1,2,3-triazole, Click Chemistry, diterpenoids, antiinflammatory activity

\section{Introduction}

The rational use of plant metabolites as a platform for the discovery of new medicinal agents takes advantage of the structurally unique building blocks provided by nature for the rapid increase of molecular complexity. Diterpene resin acids (DRA), produced by conifers, combine an unusual stereochemically defined hydrocarbon scaffold with the possibility for possible functionalization via reactions at the carboxyl group. Levopimaric acid (LpA), the main component of pine oleoresin, is one of the most important in this family.
\end{abstract}




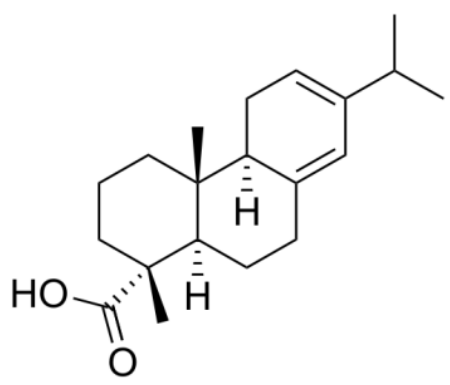

The relative content of Levopimaric acid in the resin of Scots pine Pinus sylvestris and its relative Pinus hamata reaches $27 \%$ and $36 \%$, respectively. ${ }^{1,2}$ Along with the other diterpene resin acids, it plays a key role in providing defense against insects and microbial pathogens. ${ }^{3}$ In general, the abietene types of diterpene resin acid display a broad spectrum of biological activity. In particular, derivatives of Levopimaric acid show antibacterial, cardiovascular and antioxidant activities. ${ }^{1}$

The general mode of biological activity of abietene derivatives is related to their antiinflammatory properties. These properties are based on the suppression of inflammatory cytokines production and COX-2 expression. ${ }^{4}$ Dehydroabietic acid significantly decreases the production of pro-inflammatory mediators (MCP-1, TNFó, NO) in LPS-stimulated RAW264 macrophages and in mixed cell line of macrophages and adiposytes. ${ }^{5}$ Abietic acid was used as a building block for the preparation of heterocyclic derivatives close in activity to prednisolone but less toxic. ${ }^{6}$

Synthetic transformations based on the natural compounds expand medicinal potential of the nature's molecular treasury. For compounds of levopimaric acid family, an important role is played by the Diels-Alder reaction, usually with various benzo- and naphthoquinones. This efficient transformation provides a very convenient method for the isolation of LpA from complex mixture of product derived from the pine oleoresin. Furthermore, the recently reported adducts of LpA with benzoquinones display activity comparable with orthophen. ${ }^{7}$ The derivatives of dihydroquinopimaric acid show a particularly attractive combination of high antiinflammatory activity with low toxicity. ${ }^{8}$

These examples illustrate the potential of abietic diterpenoids as a structural scaffold in the design of anti-inflammatory agents. In this aspect, LpA remains the less studied in this family of compounds. As the part of our ongoing research program dedicated to the investigation of plant metabolites produced by trees and herbs of Siberia, ${ }^{9,10}$ we present the first synthesis of acetylenic derivatives of LpA, selected transformations of these compounds, and studies of the antiinflammatory activity with the aim of better understanding of the structure-activity relation.

Presence of acetylenic moiety into natural ${ }^{11,12}$ and designed ${ }^{13}$ medicinal agents can play important role in the mechanism of their biological activity. Furthermore, it introduces possibilities for the further structural modifications. This strategy has been successfully applied to natural compounds of triterpenoid family. ${ }^{14,15}$ However, in the latest monograph on the 
properties of DRA, where synthesis and properties of levopimaric acid are described in a chapter with 142 references, ${ }^{1}$ data on acetylenic derivatives of this diterpene are absent.

\section{Results and Discussion}

Based on the above, we had chosen double modification of LpA using the Diels-Alder reaction with 1,4-naphthoquinone ${ }^{1}$ with following introduction of alkyne groups via the LpA carboxyl.

The first step of the sequence proceeds under very mild conditions (one week at the room temperature). The precipitated naphtholevopimaric acid $\mathbf{1}(\mathrm{NqLpA})$ is filtered to provide the $93 \%$ of the product. ${ }^{16}$ The NqLpA amides 4a-e with alkyne substituents were prepared by condensation of NqLpA acid chloride 2 with the library of aminoacetylenes 3a-e (see the SI part). We had used amides as the first set of targets based on our earlier reports of promising anti-inflammatory and anti-ulcer properties of related NqLpA amides. ${ }^{17}$

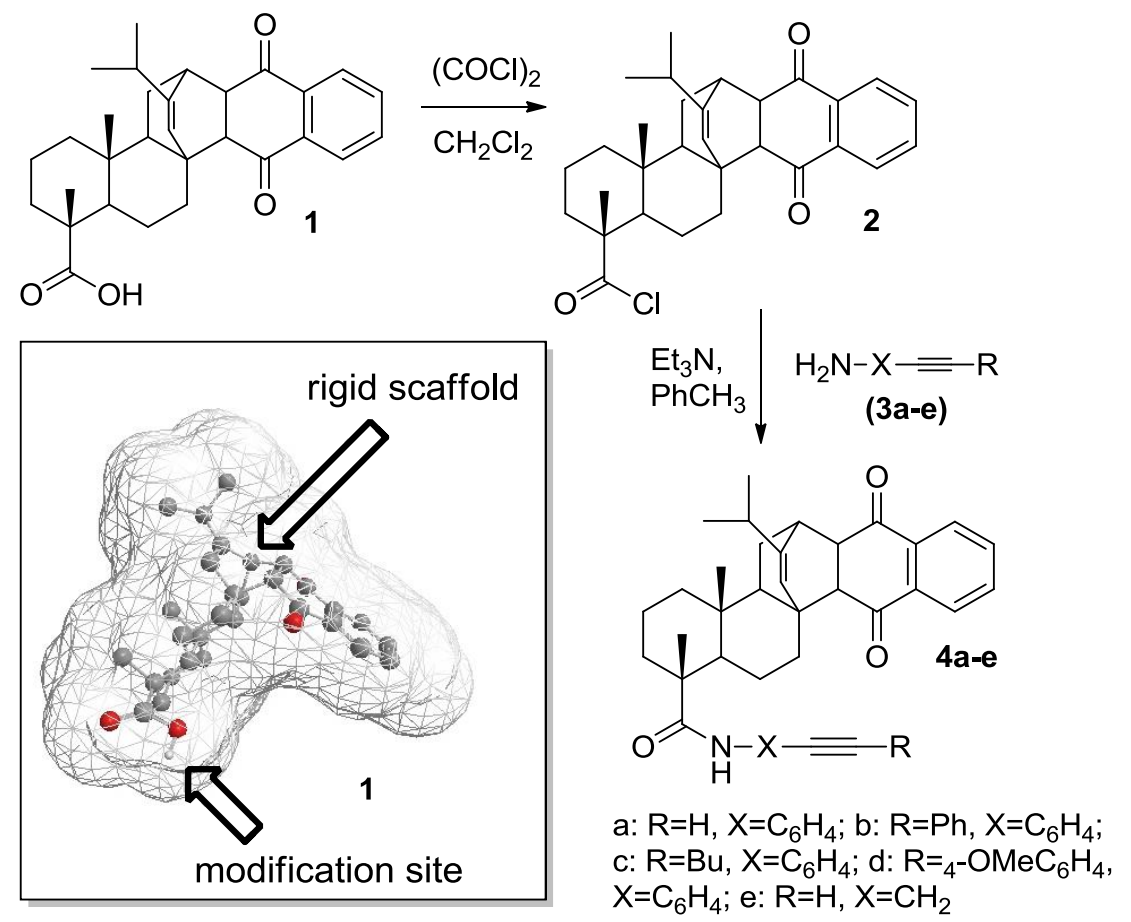

Scheme 1. Synthesis the NqLpA amides.

The NqLpA acid chloride $\mathbf{2}$ was synthesized by the reaction of acid $\mathbf{1}$ with oxalyl chloride in DCM at the room temperature. After removal of solvent from the reaction mixture, it was diluted with toluene and introduced in the reaction with the respective acetylenic amine $\mathbf{3}$ in triethylamine. 
We had also explored the possibility of modifying the acetylenic derivatives of LpA via 1,3dipolar cycloaddition with aryl and alkyl azides. The $\mathrm{Cu}$-catalyzed version of this process works very well for terminal alkynes. ${ }^{18,19}$ The high yields, attractive regioselectivity and mild conditions account for the growing popularity of this reaction (the most popular of the "click" reactions) in the preparation of bioconjugates, ${ }^{20-27}$ including those of triterpenoid family. ${ }^{15}$ This remarkable selective and cost-effective process, leading to 1,2,3-triazoles, possessing antiinflammatory activity ${ }^{28}$ prompted us to synthesize the levopimaric acid-1,2,3-triazole hybrid. The key intermediate - the propargyl ester NqLpA 5- prop-2-ynyl 16-isopropyl-1,4a-dimethyl1,2,3,4,4a,4b,5,6a,7,12,12a,13,14,14a-pentadecahydro- $\mathrm{H}$-(6,12b-etheno)benzo[ $b]$ chrysene7,12-dione-1-carboxylate 5 was obtained by the reaction of acid $\mathbf{1}$ and propargyl bromide in the presence of $\mathrm{K}_{2} \mathrm{CO}_{3}$ in $30 \%$ isolated yield.

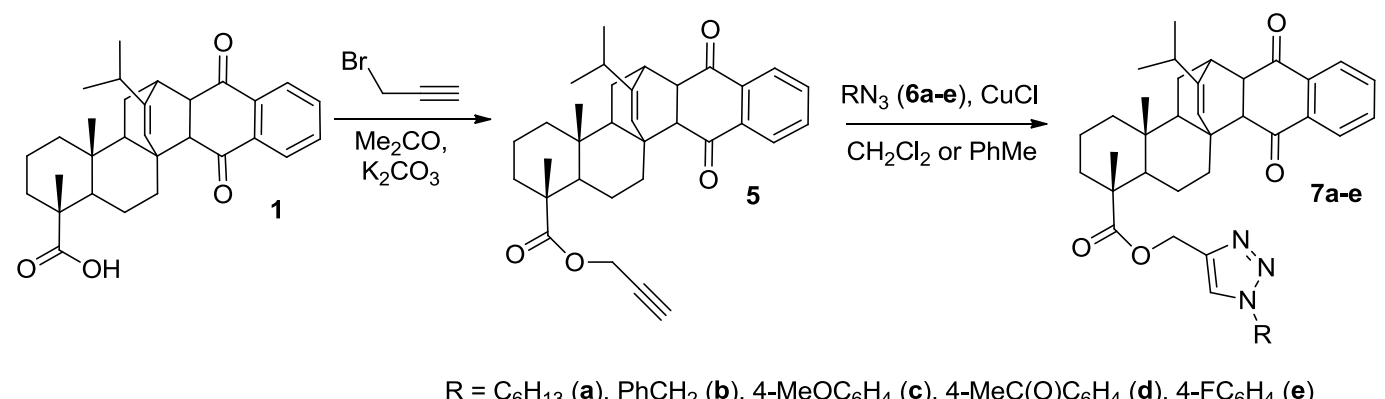

Scheme 2. Synthesis the propargyl ester NqLpA and the $1 H$-1,2,3-triazole derivatives of NqLpA.

In the following steps, the terminal alkyne 5 was introduced in the "click" reactions with alkyl and aryl azides 6a-e in the presence of $\mathrm{Cu}(\mathrm{I})$ salts. Interestingly, alkyl azides are more reactive in comparison to their aromatic analogues. Whereas the reactions with alkyl azides proceed at the room temperature in $84-96 \%$ yields, the reactions with aromatic azides require reflux in toluene ( $1 H$-1,2,3-triazole yields of $\sim 70 \%)$.

The structure of all new acetylenes and the products of their transformations was established on the basis of analytical and spectroscopical methods (IR, ${ }^{1} \mathrm{H}$ and ${ }^{13} \mathrm{C}$ NMR)

\section{Anti-inflammatory activity of levopimaric acid derivatives}

Two groups of levopimaric acid derivatives - amides with acetylenic moiety (4-5) and triazolyl esters (7) - were tested for their anti-inflammatory activities and structure-activity relationships

using the histamine-induced paw edema model. Data in Table 1 illustrate that all synthesized derivatives injected intraperitoneally in mice $(20 \mathrm{mg} / \mathrm{kg}$ dose) decrease paw edema by $23-47$ percent relatively to control group. The same effect of indomethacin is 49 percent. The majority of the levopimaric acid derivatives (4a-c, 4e, 7a-d) have moderate anti-inflammatory activity 1.5-2.0-fold lower than that of indomethacin. However, the activity of several esters $(\mathbf{4 d}, \mathbf{5}, \mathbf{7 e})$ compares favorably with that of reference drug. 
Table 1. Anti-inflammatory activity of levopimaric acid derivatives in the histamine-induced paw edema model

\begin{tabular}{|c|c|c|c|}
\hline Substance & $\begin{array}{l}\text { Inflammation index } \\
(\%)\end{array}$ & $\begin{array}{l}\text { Anti-inflammatory } \\
\text { activity }(\%)\end{array}$ & $\begin{array}{l}\text { Anti-inflammatory } \\
\text { activity relative to } \\
\text { indomethacin }(\%)\end{array}$ \\
\hline Control & $34.2 \pm 2.2^{\S \S \S}$ & 0 & - \\
\hline $4 a$ & $24.9 \pm 1.9^{*} \S \S \S$ & $27.2^{*}$ & $55.4^{\S \S \S}$ \\
\hline $4 b$ & $25.2 \pm 2.0 * \S \S \S$ & $26.3^{*}$ & $53.6^{\S \S \S}$ \\
\hline $4 c$ & $22.4 \pm 2.0 * * \S$ & $34.5^{* *}$ & $70.3^{\S}$ \\
\hline $4 d$ & $19.3 \pm 1.8^{* * *}$ & $43.6^{* * *}$ & 88.8 \\
\hline $4 e$ & $26.3 \pm 3.0 * \S \S$ & $23.1 *$ & $47.0^{\S \S}$ \\
\hline 5 & $20.1 \pm 1.3^{* * *}$ & $41.2^{* * *}$ & 83.9 \\
\hline $7 a$ & $25.9 \pm 2.1 * \S \S \S$ & $24.3^{*}$ & $49.5^{\S \S \S}$ \\
\hline $7 b$ & $22.0 \pm 1.3 * * * \S \S$ & $35.7^{* * *}$ & $72.7^{\S \S}$ \\
\hline $7 c$ & $25.4 \pm 2.0 * \S \S \S$ & $25.7^{*}$ & $52.3^{\S \S \S}$ \\
\hline 7d & $23.2 \pm 1.3 * * \S \S \S$ & $32.2 * *$ & $65.6^{\S \S \S}$ \\
\hline $7 e$ & $20.9 \pm 1.5^{* * *}$ & $38.9 * * *$ & 79.2 \\
\hline Indomethacin & $17.4 \pm 0.9 * * *$ & $49.1 * * *$ & 100 \\
\hline
\end{tabular}

$* \mathrm{P}<0.05,{ }^{*} * \mathrm{P}<0.01, * * * \mathrm{P}<0.001$ relative to control

${ }^{\S} \mathrm{P}<0.05,{ }^{\S} \mathrm{P}<0.01,{ }^{\S \S} \mathrm{P}<0.001$ relative to indomethacin

Comparative analysis of anti-inflammatory potencies of derivatives with acetylenic moiety in side residue $(\mathbf{4 a}, \mathbf{5}, \mathbf{4 e})$ indicates that it depends on the structure of the linker at carbon group. Exchange of amide moiety to ester in the side residue causes significant 1.8 fold-increase of the anti-inflammatory effect (compound 5). Introduction of a methoxy group in the diphenylacetylenic residue causes 1.7 - fold increase of activity $(\mathbf{4 d} \rightarrow \mathbf{4 b})$.

The activity of triazolic esters is also modulated by the substitution. For example, both change from an aliphatic to an aromatic substituents and fluorine introduction in the pendant aryl increase the activity. These data indicates that anti-inflammatory activity of amides and esters of LpA is sensitive to the nature of substitution and confirms the potential value of this class of biological active substances. 


\section{Conclusions}

In conclusion, we reported the first synthesis of acetylene derivatives of $\mathrm{LpA}$, their $\mathrm{Cu}$-catalyzed alkyne-azide cycloadditions reactions, and anti-inflammatory activity of the new compounds. The possibility of combining the stereochemically defined polycyclic diterpene scaffold with the flexibility of structural "click" modifications should open new opportunities for the rational design of terpene-based medicinal agents. Encouragingly, the synthesized LpA derivatives possess anti-inflammatory activity comparable to the activity of indomethacin.

\section{Experimental Section}

General. Melting points were determined with a Kofler apparatus. Column chromatography was performed on $\mathrm{Al}_{2} \mathrm{O}_{3}$ ("Aldrich") and the Silufol UV-254 plates were used for TLC analysis. The IR-spectra were recorded in $\mathrm{KBr}$ pellets on a "Bruker IFS 66" instrument. $\mathrm{PdCl}_{2}\left(\mathrm{PPh}_{3}\right)_{2}$, propargylamine, phenylacetylene, 4-iodaniline, $\mathrm{Et}_{3} \mathrm{~N}, \mathrm{CuCl}$ were commercially available reactants ("Aldrich"). All the organic solvents were of analytical quality. Combustion analysis was performed with CHN-analyzer (Model 1106, "Carlo Erba", Italy). NMR spectra were recorded on a «Bruker AM-400» $400.13\left({ }^{1} \mathrm{H}\right)$ and $100.61 \mathrm{MHz}\left({ }^{13} \mathrm{C}\right)$ at $25^{\circ} \mathrm{C}$.

16-Isopropyl-1,4a-dimethyl-1,2,3,4,4a,4b,5,6a,7,12,12a,13,14,14a-pentadecahydro-2H$(6,12 b-e t h e n o) b e n z o[b]$ chrysene-7,12-dione-1-carboxylic acid (1) was prepared as described in ref.16.

General procedure of syntheses of aminoacetylenes 3. A mixture of 4-iodaniline (3mmol), corresponding alkyne-1 $(3.2 \mathrm{mmol}), \mathrm{PdCl}_{2}\left(\mathrm{PPh}_{3}\right)_{2}(10 \mathrm{mg}), \mathrm{CuI}(5 \mathrm{mg})$ and $\mathrm{Et}_{3} \mathrm{~N}(2 \mathrm{~mL})$ in benzene $(25 \mathrm{~mL})$ was stirred at $70{ }^{\circ} \mathrm{C}$ under the argon atmosphere. When the coupling was complete (TLC control) the products 3 was separated by column chromatography using hexane and then hexane-ethylacetate mixtures as eluent and recrystallized. Spectroscopic data and melting points for 4-(p-methoxyphenyl)ethynylaniline (3d) ${ }^{29}$ 4-phenylethynylaniline (3b) ${ }^{30} n$ butylethynylaniline (3c) ${ }^{31}$ and $p$-aminophenylacetylene (3a) ${ }^{32}$ were consistent with the literature data.

General procedure of syntheses of amides 4. A mixture of 16-isopropyl-1,4a-dimethyl1,2,3,4,4a,4b,5,6a,7,12,12a,13,14,14a-pentadecahydro- $2 \mathrm{H}$-(6,12b-etheno)benzo[ $b]$ chrysene-

7,12-dione-1-carboxylic acid $1(500 \mathrm{mg}, 1.0 \mathrm{mmol})$ and oxalyl chloride $(0.3 \mathrm{~mL}, 4.3 \mathrm{mmol})$ in of dry dichloromethane $(10 \mathrm{~mL})$ was stirred in argon for $3.5 \mathrm{~h}$ at room temperature. The solvent was evaporated at a reduced pressure. This crude product $\mathbf{2}$ without further purification was dissolved in of toluene $(10 \mathrm{~mL})$ and added to mixture $\mathrm{Et}_{3} \mathrm{~N}(0.25 \mathrm{~mL})$ and amine $3(1.1 \mathrm{mmol})$ in of toluene $(5 \mathrm{ml})$. A mixture was stirred for $1-4 \mathrm{~h}$ at $75-80^{\circ} \mathrm{C}$ under the argon atmosphere. The solvent was evaporated at a reduced pressure, the product $\mathbf{4}$ was purified by chromatography on 
$\mathrm{Al}_{2} \mathrm{O}_{3}$ (elution with dichloromethane and mixture ethyl acetate - toluene) and crystallized from hexane.

$N$-(4-Ethynylphenyl)-16-isopropyl-1,4a-dimethyl-1,2,3,4,4a,4b,5,6a,7,12,12a,13,14,14apentadecahydro- $2 H$-(6,12b-etheno)benzo- $[\boldsymbol{b}]$ chrysene-7,12-dione-1-carboxamide (4a). The yield is $224 \mathrm{mg}(40 \%), \mathrm{mp} 150-153{ }^{\circ} \mathrm{C}$ (hexane); IR $\left(\mathrm{KBr}, \mathrm{v}, \mathrm{cm}^{-1}\right)$ : 1680, $1726(\mathrm{C}=\mathrm{O}) ; 2106$ $(\mathrm{C} \equiv \mathrm{C}) .{ }^{1} \mathrm{H}$ NMR $\left(400 \mathrm{MHz}, \mathrm{CDCl}_{3}\right): \delta 0.49(3 \mathrm{H}, \mathrm{d}, J 6.7 \mathrm{~Hz}), 0.58(3 \mathrm{H}, \mathrm{s}), 0.86(3 \mathrm{H}, \mathrm{d}, J 6.7$ $\mathrm{Hz}), 0.90(1 \mathrm{H}, \mathrm{m}), 1.26(3 \mathrm{H}, \mathrm{s}), 1.37(2 \mathrm{H}, \mathrm{m}), 1.49-1.60(6 \mathrm{H}, \mathrm{m}), 1.65(1 \mathrm{H}, \mathrm{m}), 1.76-1.93(5 \mathrm{H}$, m), $2.85(1 \mathrm{H}, \mathrm{d}, J 8.6 \mathrm{~Hz}), 3.06(1 \mathrm{H}, \mathrm{s}), 3.07(1 \mathrm{H}, \mathrm{br} . \mathrm{s}), 3.25(1 \mathrm{H}, \mathrm{dd}, J 3.0,8.6 \mathrm{~Hz}), 5.06(1 \mathrm{H}$, s), 7.44-7.54 (5H, m), $7.60(2 \mathrm{H}, \mathrm{m}), 7.69(1 \mathrm{H}, \mathrm{m}), 7.78(1 \mathrm{H}, \mathrm{m}) .{ }^{13} \mathrm{C} \mathrm{NMR}\left(100 \mathrm{MHz}, \mathrm{CDCl}_{3}\right): \delta$ $16.37,17.07,17.30,19.13,20.73,21.73,28.19,33.24,35.19,37.15,37.75,38.53,42.21,42.38$, 47.93, 50.33, 52.17, 56.37, 59.35, 77.36, 83.57, 117.82, 119.83, 125.13, 125.92, 125.96, 133.08, 133.24, 134.02, 136.85, 138.47, 138.64, 147.71, 176.83, 198.28, 198.80. Anal. Calcd for $\mathrm{C}_{38} \mathrm{H}_{41} \mathrm{NO}_{3}$ : C, 81.54; H, 7.38; N, 2.50\%. Found: C, 81.23; H, 7.49; N, 2.65\%.

$\mathrm{N}$-(4-(Phenylethynyl)phenyl)-16-isopropyl-1,4a-dimethyl-

1,2,3,4,4a,4b,5,6a,7,12,12a,13,14,14a-pentadecahydro-2H-(6,12b-etheno)benzo-[b]chrysene7,12-dione-1-carboxamide (4b). The yield is $204 \mathrm{mg}(32 \%), \mathrm{mp} 149-151^{\circ} \mathrm{C}$ (hexane); IR ( $\mathrm{KBr}$, $\left.v, \mathrm{~cm}^{-1}\right): 1680(\mathrm{C}=\mathrm{O}) ; 2214(\mathrm{C} \equiv \mathrm{C}) .{ }^{1} \mathrm{H} \mathrm{NMR}\left(\mathrm{CDCl}_{3}, 400 \mathrm{MHz}\right): \delta 0.50(3 \mathrm{H}, \mathrm{d}, J 6.7 \mathrm{~Hz}), 0.58$ $(3 \mathrm{H}, \mathrm{s}), 0.87(3 \mathrm{H}, \mathrm{d}, J 6.7 \mathrm{~Hz}), 1.01(1 \mathrm{H}, \mathrm{m}), 1.27(3 \mathrm{H}, \mathrm{s}), 1.29-1.58(8 \mathrm{H}, \mathrm{m}), 1.66(1 \mathrm{H}, \mathrm{m}), 1.78-$ $1.92(5 \mathrm{H}, \mathrm{m}), 2.86(1 \mathrm{H}, \mathrm{d}, J 8.6 \mathrm{~Hz}), 3.07(1 \mathrm{H}, \mathrm{br} . \mathrm{s}), 3.25(1 \mathrm{H}, \mathrm{dd}, J 2.9,8.6 \mathrm{~Hz}), 5.07(1 \mathrm{H}, \mathrm{s})$, $7.34(3 \mathrm{H}, \mathrm{m}), 7.45-7.57(7 \mathrm{H}, \mathrm{m}), 7.60(2 \mathrm{H}, \mathrm{m}), 7.70(1 \mathrm{H}, \mathrm{m}), 7.79(1 \mathrm{H}, \mathrm{m}) .{ }^{13} \mathrm{C} \mathrm{NMR}(100 \mathrm{MHz}$, $\left.\mathrm{CDCl}_{3}\right): \delta 16.38,17.09,17.31,19.13,20.74,21.74,28.19,33.24,35.20,37.17,37.76,38.53$, $42.22,42.39,47.92,50.33,52.18,56.37,59.35,89.15,89.35,119.06,119.91,123.47,125.14$, $125.92,125.96,128.29,128.48,131.69,132.53,133.24,134.02,136.85,138.01,138.65,147.70$, 176.80, 198.30, 198.82. Anal. Calcd for $\mathrm{C}_{44} \mathrm{H}_{45} \mathrm{NO}_{3}$ : C, 83.11; H, 7.13; N, 2.20\%. Found: C, $83.11 ; \mathrm{H}, 7.13 ; \mathrm{N}, 2.64 \%$.

N-(4-(Hex-1-ynyl)phenyl)-16-isopropyl-1,4a-dimethyl-1,2,3,4,4a,4b,5,6a,7,12,12a,13,14,14apentadecahydro- $2 \mathrm{H}$-(6,12b-etheno)benzo- $[\boldsymbol{b}]$ chrysene-7,12-dione-1-carboxamide (4c). The yield is $205 \mathrm{mg}(33 \%), \mathrm{mp} 121-123{ }^{\circ} \mathrm{C}$ (hexane); $\mathrm{IR}\left(\mathrm{KBr}, \mathrm{v}, \mathrm{cm}^{-1}\right): 1680(\mathrm{C}=\mathrm{O}) ; 2230(\mathrm{C} \equiv \mathrm{C})$.

${ }^{1} \mathrm{H}$ NMR (400 MHz, $\left.\mathrm{CDCl}_{3}\right): \delta 0.50(3 \mathrm{H}, \mathrm{d}, J 6.7 \mathrm{~Hz}), 0.58(3 \mathrm{H}, \mathrm{s}), 0.86(3 \mathrm{H}, \mathrm{d}, J 6.7 \mathrm{~Hz}), 0.96$ $(3 \mathrm{H}, \mathrm{t}, J 7.3 \mathrm{~Hz}), 1.04(1 \mathrm{H}, \mathrm{dd}, J 6.7,12.4 \mathrm{~Hz}), 1.25(3 \mathrm{H}, \mathrm{s}), 1.28-1.68(13 \mathrm{H}, \mathrm{m}), 1.78-1.91(5 \mathrm{H}$, m), $2.41(2 \mathrm{H}, \mathrm{t}, J 7.0 \mathrm{~Hz}), 2.86(1 \mathrm{H}, \mathrm{d}, J 8.6 \mathrm{~Hz}), 3.06(1 \mathrm{H}, \mathrm{br}), 3.25(1 \mathrm{H}, \mathrm{dd}, J 2.9,8.6 \mathrm{~Hz}), 5.06$ $(1 \mathrm{H}, \mathrm{s}), 7.37(2 \mathrm{H}, \mathrm{m}), 7.41(1 \mathrm{H}, \mathrm{s}), 7.47(2 \mathrm{H}, \mathrm{m}), 7.60(2 \mathrm{H}, \mathrm{m}), 7.69(1 \mathrm{H}, \mathrm{m}), 7.78(1 \mathrm{H}, \mathrm{m}) .{ }^{13} \mathrm{C}$ NMR (100 MHz, CDCl3): $\delta$ 13.82, 16.36, 17.08, 17.31, 19.13, 19.28, 20.73, 21.72, 33.19, 28.21, 31.02 , 33.24, 35.20, 37.16, 37.77, 38.53, 42.21, 42.39, 47.85, 50.30, 52.19, 56.37, 59.36, 80.35, $90.10,119.88,120.02,125.18,125.92,125.96,132.37,133.22,134.01,136.86,137.29,138.67$, 147.69, 176.69, 198.27, 198.82. HRMS: $m / z[\mathrm{M}-2 \mathrm{H}]^{+}$calcd 615.3707 , found 613.3550 for $\mathrm{C}_{42} \mathrm{H}_{49} \mathrm{NO}_{3}$.

$\mathrm{N}$-(4-(4-Methoxyphenylethynyl)phenyl)-16-isopropyl-1,4a-dimethyl1,2,3,4,4a,4b,5,6a,7,12,12a,13,14,14a-pentadecahydro- $2 H$-(6,12b-etheno)-benzo[b]chrysene7,12-dione-1-carboxamide (4d). The yield is $240 \mathrm{mg}(36 \%), \mathrm{mp} 149-151^{\circ} \mathrm{C}$ (hexane); $\mathrm{IR}(\mathrm{KBr}$, 
$\left.v, \mathrm{~cm}^{-1}\right): 1680(\mathrm{C}=\mathrm{O}) ; 2214(\mathrm{C} \equiv \mathrm{C}) .{ }^{1} \mathrm{H}$ NMR $\left(400 \mathrm{MHz}, \mathrm{CDCl}_{3}\right): \delta 0.50(3 \mathrm{H}, \mathrm{d}, J 6.7 \mathrm{~Hz}), 0.58$ $(3 \mathrm{H}, \mathrm{s}), 0.86(3 \mathrm{H}, \mathrm{d}, J 6.7 \mathrm{~Hz}), 1.03(1 \mathrm{H}, \mathrm{m}), 1.26(3 \mathrm{H}, \mathrm{s}), 1.28-1.58(8 \mathrm{H}, \mathrm{m}), 1.65(1 \mathrm{H}, \mathrm{m}), 1.78-$ $1.91(5 \mathrm{H}, \mathrm{m}), 2.86(1 \mathrm{H}, \mathrm{d}, J 8.6 \mathrm{~Hz}), 3.07(1 \mathrm{H}, \mathrm{br}), 3.25(1 \mathrm{H}, \mathrm{dd}, J 2.9,8.6 \mathrm{~Hz}), 3.83(3 \mathrm{H}, \mathrm{s})$, $5.06(1 \mathrm{H}, \mathrm{s}), 6.88(2 \mathrm{H}, \mathrm{m}), 7.45-7.56(7 \mathrm{H}, \mathrm{m}), 7.60(2 \mathrm{H}, \mathrm{m}), 7.69(1 \mathrm{H}, \mathrm{m}), 7.78(1 \mathrm{H}, \mathrm{m}) .{ }^{13} \mathrm{C}$ NMR (100 MHz, $\left.\mathrm{CDCl}_{3}\right): \delta 16.37,17.08,17.31,19.13,20.73,21.74,28.20,33.23,35.21,37.17$, 37.77, 38.53, 42.21, 42.39, 47.89, 50.31, 52.18, 55.44, 56.37, 59.35, 88.02, 89.12, 114.13, $115.59,119.43,119.94,125.16,125.92,125.95,132.33,133.13,133.21,134.00,136.85,137.73$, 138.65, 147.69, 159.67, 176.76, 198.27, 198.80. Anal. Calcd for $\mathrm{C}_{45} \mathrm{H}_{47} \mathrm{NO}_{4}$ : C, 81.17; H, 7.11; N, 2.10\%. Found: C, 81.12; H, 7.03; N, 2.19\%.

\section{$N$-Prop-2-ynyl-16-isopropyl-1,4a-dimethyl-1,2,3,4,4a,4b,5,6a,7,12,12a,13,14,14a-} pentadecahydro- $2 H$-(6,12b-etheno)-benzo $[b]$ chrysene-7,12-dione-1-carboxamide (4e). The yield is $120 \mathrm{mg}(24 \%), \mathrm{mp} 110-112^{\circ} \mathrm{C}$ (hexane); IR (KBr, v, $\left.\mathrm{cm}^{-1}\right): 1661,1678(\mathrm{C}=\mathrm{O}) ; 2120$, $3310(\mathrm{C} \equiv \mathrm{CH}) .{ }^{1} \mathrm{H}$ NMR $\left(400 \mathrm{MHz}, \mathrm{CDCl}_{3}\right): \delta 0.49(3 \mathrm{H}, \mathrm{d}, J 6.5 \mathrm{~Hz}), 0.54(3 \mathrm{H}, \mathrm{s}), 0.85(3 \mathrm{H}, \mathrm{d}, J$ $6.5 \mathrm{~Hz}), 1.04(1 \mathrm{H}, \mathrm{dd}, J 6.7,11.6 \mathrm{~Hz}), 1.13(3 \mathrm{H}, \mathrm{s}), 1.21-1.71(9 \mathrm{H}, \mathrm{m}), 1.75-1.89(5 \mathrm{H}, \mathrm{m}), 2.25$ $(1 \mathrm{H}, \mathrm{s}), 2.84$ (1H, d, J 8.6 Hz), 3.05 (1H, br.s), 3.24 (1H, dd, J 2.7, 8.6 Hz), 4.05 (2H, m), 5.05 $(1 \mathrm{H}, \mathrm{s}), 5.87\left(1 \mathrm{H}\right.$, br.s), $7.60(2 \mathrm{H}, \mathrm{m}), 7.70(1 \mathrm{H}, \mathrm{m}), 7.78(1 \mathrm{H}, \mathrm{m}) .{ }^{13} \mathrm{C} \mathrm{NMR}\left(100 \mathrm{MHz}, \mathrm{CDCl}_{3}\right)$ : $\delta 16.33,16.78,17.22,19.12,20.72,21.53,28.22,29.79,33.22,35.11,36.93,37.76,38.46,42.23$, $42.38,46.89,50.13,52.20,56.23,59.38,71.80,80.00,125.22,125.92,125.93,133.20,133.98$, 136.87, 138.71, 147.60, 178.24, 198.31, 198.86. Anal. Calcd for $\mathrm{C}_{33} \mathrm{H}_{39} \mathrm{NO}_{3}: \mathrm{C}, 79.64 ; \mathrm{H}, 7.90$; N, 2.81\%. Found: C, 79.52; H, 8.34; N, 2.78\%.

\section{Prop-2-ynyl-16-isopropyl-1,4a-dimethyl-1,2,3,4,4a,4b,5,6a,7,12,12a,13,14,14a-} pentadecahydro- $2 H$-(6,12b-etheno)benzo $[b]$ chrysene-7,12-dione-1-carboxylate (5). A mixture of 16-isopropyl-1,4a-dimethyl-1,2,3,4,4a,4b,5,6a,7,12,12a,13,14,14a-pentadecahydro2H-(6,12b-etheno)benzo[b]chrysene-7,12-dione-1-carboxylic acid 1 (7 g, $15.2 \mathrm{mmol})$, propargyl bromide (3.6 g, $30.4 \mathrm{mmol})$ and $\mathrm{K}_{2} \mathrm{CO}_{3}(4.8 \mathrm{~g}, 45.6 \mathrm{mmol})$ in acetone $(190 \mathrm{~mL})$ was boiled for 7 h. Then mixture was filtered, the solvent was evaporated at a reduced pressure. The crude product was purified by column chromatography on $\mathrm{Al}_{2} \mathrm{O}_{3}$ (elution with toluene and dichloromethane). The yield is $2.3 \mathrm{~g}(30 \%), \mathrm{mp} 175-176{ }^{\circ} \mathrm{C}$ (hexane); IR (KBr, $\left.v, \mathrm{~cm}^{-1}\right): 1680$, $1722(\mathrm{C}=\mathrm{O}) ; 2131,3290(\mathrm{C} \equiv \mathrm{CH}) .{ }^{1} \mathrm{H}$ NMR (400 MHz, $\left.\mathrm{CDCl}_{3}\right): \delta 0.49(3 \mathrm{H}, \mathrm{d}, J 6.7 \mathrm{~Hz}), 0.53$ $(3 \mathrm{H}, \mathrm{s}), 0.85(3 \mathrm{H}, \mathrm{d}, J 6.7 \mathrm{~Hz}), 0.94(1 \mathrm{H}, \mathrm{m}), 1.14(3 \mathrm{H}, \mathrm{s}), 1.25(2 \mathrm{H}, \mathrm{m}), 1.35-1.58(6 \mathrm{H}, \mathrm{m}), 1.65$ $(1 \mathrm{H}, \mathrm{m}), 1.72-1.90(5 \mathrm{H}, \mathrm{m}), 2.48(1 \mathrm{H}, \mathrm{t}, J 2.4 \mathrm{~Hz}), 2.87(1 \mathrm{H}, \mathrm{d}, J 8.6 \mathrm{~Hz}), 3.05(1 \mathrm{H}, \mathrm{br}(\mathrm{m})), 3.25$ $(1 \mathrm{H}, \mathrm{dd}, J 2.9,8.6 \mathrm{~Hz}), 4.68(2 \mathrm{H}, \mathrm{m}), 5.06(1 \mathrm{H}, \mathrm{br} . \mathrm{s}), 7.61(2 \mathrm{H}, \mathrm{m}), 7.71(1 \mathrm{H}, \mathrm{m}), 7.79(1 \mathrm{H}, \mathrm{m})$. ${ }^{13} \mathrm{C}$ NMR $\left(100 \mathrm{MHz}, \mathrm{CDCl}_{3}\right): \delta 16.23,16.82,17.10,19.11,20.73,22.07,28.21,33.24,35.02$, $36.48,37.82,38.33,42.24,42.45,47.28,49.35,52.23,52.26,56.23,59.41,74.75,78.10,125.19$, $125.90,125.93,133.26,134.02,136.91,138.70,147.60,177.93,198.57,198.86$. Anal. Calcd for $\mathrm{C}_{33} \mathrm{H}_{38} \mathrm{O}_{4}$ : C, 79.48; H, 7.68\%. Found: C, 79.69; H, 7.62\%.

General procedure of syntheses of arylazides 6c-e. To a solution of amine in THF (10-20 mL) was added $\mathrm{HCl}(1-6 \mathrm{~mL})$ and water $(8-47 \mathrm{~mL})$. The reaction mixture was stirred for $15 \mathrm{~min}$ at 0 ${ }^{\circ} \mathrm{C}$, and a solution of $\mathrm{NaNO}_{2}(4.4-29 \mathrm{mmol})$ in of water $(3-23 \mathrm{~mL})$ was added dropwise to the solution, keeping the temperature of the reaction mixture below $5{ }^{\circ} \mathrm{C}$. The reaction mixture was 
stirred for $15 \mathrm{~min}$ at $0{ }^{\circ} \mathrm{C}$, and then to the cooled reaction mixture was added dropwise a solution of $\mathrm{NaN}_{3}(5.5-36 \mathrm{mmol})$ in of water $(4-23 \mathrm{~mL})$. After the addition, the reaction mixture was stirred for $1 \mathrm{~h}$ at room temperature. The organic layer was extracted with dichloromethane, and the extract was washed with dilute hydrochloric acid $(0.1 \mathrm{M})$ and dried over $\mathrm{Na}_{2} \mathrm{SO}_{4}$. The solvent was removed in vacuo. Hexane was added and the resulting precipitate was filtered off to afford of the azide.

4-Azidoanisole (6c), ${ }^{33}$ p-azidoacetophenone (6d), ${ }^{34}$ 1-azido-4-fluorobenzene $(\mathbf{6 e}),{ }^{35} n$-hexyl azide (6a) ${ }^{36}$ benzyl azide $(\mathbf{6 b})^{37}$ were prepared as described in the literature.

Syntheses of triazoles. Method A. A mixture of prop-2-ynyl-16-isopropyl-1,4a-dimethyl1,2,3,4,4a,4b,5,6a,7,12,12a,13,14,14a-pentadecahydro- $2 H$-(6,12b-etheno)benzo[ $b]$ chrysene-

7,12-dione-1-carboxylate 5 (200 mg, $0.4 \mathrm{mmol}), \mathrm{CuCl}(4 \mathrm{mg}, 0.04 \mathrm{mmol})$ and alkyl azide 6a,b $(0.4 \mathrm{mmol})$ in dichloromethane $(4 \mathrm{~mL})$ was stirred at room temperature for $4-5 \mathrm{~h}$. The crude product was purified by chromatography on silica gel or $\mathrm{Al}_{2} \mathrm{O}_{3}$ (elution with dichloromethane and ethyl acetate) and crystallized from hexane.

\section{(1-Hexyl-1H-1,2,3-triazol-4-yl)methyl-16-isopropyl-1,4a-dimethyl-}

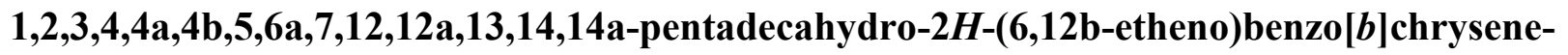
7,12-dione-1-carboxylate (7a). The yield is $240 \mathrm{mg}(96 \%), \mathrm{mp} 52-55^{\circ} \mathrm{C}$ (hexane); $\mathrm{IR}(\mathrm{KBr}, \mathrm{v}$, $\left.\mathrm{cm}^{-1}\right)$ : 1678, $1722(\mathrm{C}=\mathrm{O}) .{ }^{1} \mathrm{H}$ NMR $\left(400 \mathrm{MHz}, \mathrm{CDCl}_{3}\right) \delta 0.48(3 \mathrm{H}, \mathrm{d}, J 6.7 \mathrm{~Hz}), 0.51(3 \mathrm{H}, \mathrm{s})$, $0.84(3 \mathrm{H}, \mathrm{d}, J 6.7 \mathrm{~Hz}), 0.86-0.91(4 \mathrm{H}, \mathrm{m}), 1.11(3 \mathrm{H}, \mathrm{s}), 1.19-1.96(22 \mathrm{H}, \mathrm{m}), 2.84(1 \mathrm{H}, \mathrm{d}, J 8.6$ Hz), 3.04 (1H, br.s), 3.23 (1H, dd, J 2.7, 8.6 Hz), $4.40(2 \mathrm{H}, \mathrm{t}, J 7.2 \mathrm{~Hz}), 5.04(1 \mathrm{H}, \mathrm{s}), 5.24(1 \mathrm{H}, \mathrm{q}$, $J$ 12.5, $25.7 \mathrm{~Hz}), 7.60(3 \mathrm{H}, \mathrm{m}), 7.69(1 \mathrm{H}, \mathrm{m}), 7.78(1 \mathrm{H}, \mathrm{m}) .{ }^{13} \mathrm{C} \mathrm{NMR}\left(100 \mathrm{MHz}, \mathrm{CDCl}_{3}\right): \delta$ $14.12,16.19,16.78,17.05,19.10,20.71,22.07,22.60,26.30,28.18,30.38,31.29,33.23,35.09$, $36.55,37.77,38.30,42.22,42.38,47.17,49.41,50.58,52.18,56.23,58.02,59.38,123.64$, 125.11, 125.87, 125.93, 133.26, 133.99, 136.87, 138.65, 143.25, 147.63, 178.73, 198.40, 198.86. Anal. Calcd for $\mathrm{C}_{39} \mathrm{H}_{51} \mathrm{~N}_{3} \mathrm{O}_{4}$ : C, 74.85; H, 8.21; N, 6.71\%. Found: C, 74.80; H, 8.11; N, 6.81\%.

\section{(1-Benzyl-1H-1,2,3-triazol-4-yl)methyl-16-isopropyl-1,4a-dimethyl-}

\section{1,2,3,4,4a,4b,5,6a,7,12,12a,13,14,14a-pentadecahydro- $2 H-(6,12 b$-etheno $)$ benzo $[b]$ chrysene-}

7,12-dione-1-carboxylate (7b). The yield is $210 \mathrm{mg}(84 \%), \mathrm{mp} 72-74{ }^{\circ} \mathrm{C}$ (hexane); $\mathrm{IR} \mathrm{(KBr,} v$, $\left.\mathrm{cm}^{-1}\right)$ : 1678, $1722(\mathrm{C}=\mathrm{O}) .{ }^{1} \mathrm{H} \mathrm{NMR}\left(400 \mathrm{MHz}, \mathrm{CDCl}_{3}\right): \delta 0.48(3 \mathrm{H}, \mathrm{d}, J 6.9 \mathrm{~Hz}), 0.50(3 \mathrm{H}, \mathrm{s})$, $0.84(3 \mathrm{H}, \mathrm{d}, J 6.9 \mathrm{~Hz}), 0.91(1 \mathrm{H}, \mathrm{m}), 1.08(3 \mathrm{H}, \mathrm{s}), 1.20-1.89(14 \mathrm{H}, \mathrm{m}), 2.83(1 \mathrm{H}, \mathrm{d}, J 8.8 \mathrm{~Hz})$, $3.04(1 \mathrm{H}, \mathrm{s}), 3.23(1 \mathrm{H}, \mathrm{dd}, J 2.7,8.6 \mathrm{~Hz}), 5.03(1 \mathrm{H}, \mathrm{s}), 5.21(2 \mathrm{H}, \mathrm{q}, J 12.7,15.4 \mathrm{~Hz}), 5.60(2 \mathrm{H}$, $\mathrm{m}), 7.36(5 \mathrm{H}, \mathrm{m}), 7.52(1 \mathrm{H}, \mathrm{s}), 7.61(2 \mathrm{H}, \mathrm{m}), 7.71(1 \mathrm{H}, \mathrm{m}), 7.79(1 \mathrm{H}, \mathrm{m}) .{ }^{13} \mathrm{C} \mathrm{NMR}(100 \mathrm{MHz}$, $\left.\mathrm{CDCl}_{3}\right): \delta 16.17,16.76,17.04,19.11,20.70,22.02,28.18,33.22,35.10,36.48,37.77,38.28$, $42.21,42.38,47.15,49.40,52.17,54.35,56.20,57.90,59.38,123.69,125.11,125.87,125.95$, 128.29, 128.92, 129.29, 133.26, 134.00, 134.73, 136.88, 138.65, 143.77, 147.64, 178.63, 198.40, 198.83. Anal. Calcd for $\mathrm{C}_{40} \mathrm{H}_{45} \mathrm{~N}_{3} \mathrm{O}_{4}$ : C, 76.04; H, 7.18; N, 6.65\%. Found: C, 76.14; H, 7.47; N, $6.76 \%$.

Method B. A mixture of prop-2-ynyl-16-isopropyl-1,4a-dimethyl-1,2,3,4,4a,4b,5,6a,7,12,12a, 13,14,14a-pentadecahydro-2H-(6,12b-etheno)benzo[b]chrysene-7,12-dione-1-carboxylate 5 (200 $\mathrm{mg}, 0.4 \mathrm{mmol}), \mathrm{CuCl}(4 \mathrm{mg}, 0.04 \mathrm{mmol})$ and aryl azide $\mathbf{6 c - e}(0.4 \mathrm{mmol})$ in $6 \mathrm{~mL}$ of toluene was 
boiled for $1 \mathrm{~h}$. The product was purified by chromatography on silica gel or $\mathrm{Al}_{2} \mathrm{O}_{3}$ (elution with dichloromethane and ethyl acetate) and crystallized from hexane.

(1-(4-Methoxyphenyl)-1 H-1,2,3-triazol-4-yl)methyl-16-isopropyl-1,4a-dimethyl1,2,3,4,4a,4b,5,6a,7,12,12a,13,14,14a-pentadecahydro-2H-(6,12b-etheno)benzo[b]chrysene7,12-dione-1-carboxylate (7c). The yield is $180 \mathrm{mg}(70 \%)$, mp 91-93 ${ }^{\circ} \mathrm{C}$ (hexane); $\mathrm{IR}(\mathrm{KBr}, v$, $\left.\mathrm{cm}^{-1}\right)$ : 1678, $1722(\mathrm{C}=\mathrm{O}) .{ }^{1} \mathrm{H} \mathrm{NMR}\left(400 \mathrm{MHz}, \mathrm{CDCl}_{3}\right): \delta 0.47(3 \mathrm{H}, \mathrm{d}, J 7.0 \mathrm{~Hz}), 0.52(3 \mathrm{H}, \mathrm{s})$, $0.84(3 \mathrm{H}, \mathrm{d}, J 7.0 \mathrm{~Hz}), 0.90(1 \mathrm{H}, \mathrm{m}), 1.12(3 \mathrm{H}, \mathrm{s}), 1.25-1.86(14 \mathrm{H}, \mathrm{m}), 2.85(1 \mathrm{H}, \mathrm{d}, J 8.6 \mathrm{~Hz})$, 3.04 (1H, br.s), 3.24 (1H, dd, $J$ 2.9, $8.6 \mathrm{~Hz}), 3.88(3 \mathrm{H}, \mathrm{s}), 5.04(1 \mathrm{H}, \mathrm{s}), 5.31$ (2H, q, $J 12.6,28.5$

$\mathrm{Hz}), 7.07(2 \mathrm{H}, \mathrm{d}, J 9.13 \mathrm{~Hz}), 7.61(2 \mathrm{H}, \mathrm{m}), 7.70(3 \mathrm{H}, \mathrm{m}), 7.78(1 \mathrm{H}, \mathrm{m}), 7.96(1 \mathrm{H}, \mathrm{s}) .{ }^{13} \mathrm{C}$ NMR $\left(100 \mathrm{MHz}, \mathrm{CDCl}_{3}\right): \delta 16.22,16.82,17.06,19.10,20.71,22.16,28.18,33.23,35.18,36.62,37.79$, $38.33,42.22$, 42.43, 47.24, 49.49, 52.21, 55.79, 56.24, 58.02, 59.39, 115.00, 122.16, 122.57 , 125.12 , 125.89, 125.93, 130.55, 133.25, 134.00, 136.90, 138.69, 143.87, 147.65, 160.08, 178.77, 198.40, 198.84. Anal. Calcd for $\mathrm{C}_{40} \mathrm{H}_{45} \mathrm{~N}_{3} \mathrm{O}_{5}$ : C, 74.16; H, 7.00; N, 6.49\%. Found: C, 74.59; H, 7.32; N, 6.43\%.

(1-(4-Acetylphenyl)-1 H-1,2,3-triazol-4-yl)methyl-16-isopropyl-1,4a-dimethyl-

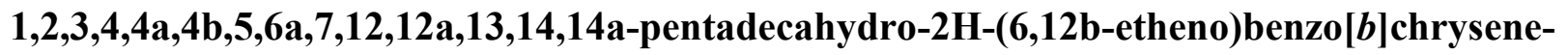
7,12-dione-1-carboxylate (7d). The yield is $184 \mathrm{mg}(70 \%), \mathrm{mp} 97-99{ }^{\circ} \mathrm{C}$ (hexane); $\mathrm{IR}(\mathrm{KBr}, v$, $\left.\mathrm{cm}^{-1}\right)$ : 1681, $1724(\mathrm{C}=\mathrm{O}) .{ }^{1} \mathrm{H} \mathrm{NMR}\left(400 \mathrm{MHz}, \mathrm{CDCl}_{3}\right): \delta 0.48(3 \mathrm{H}, \mathrm{d}, J 6.7 \mathrm{~Hz}), 0.52(3 \mathrm{H}, \mathrm{s})$, $0.85(3 \mathrm{H}, \mathrm{d}, J 6.7 \mathrm{~Hz}), 0.91(1 \mathrm{H}, \mathrm{m}), 1.13(3 \mathrm{H}, \mathrm{s}), 1.20-1.89(14 \mathrm{H}, \mathrm{m}), 2.67(3 \mathrm{H}, \mathrm{s}), 2.85(1 \mathrm{H}, \mathrm{d}$, $J 8.6 \mathrm{~Hz}), 3.05$ (1H, br.s), $3.24(1 \mathrm{H}, \mathrm{dd}, J 2.9,8.6 \mathrm{~Hz}), 5.04(1 \mathrm{H}, \mathrm{s}), 5.33(2 \mathrm{H}, \mathrm{q}, J 12.8,17.8$ $\mathrm{Hz}), 7.61(2 \mathrm{H}, \mathrm{m}), 7.68(1 \mathrm{H}, \mathrm{m}), 7.79(1 \mathrm{H}, \mathrm{m}), 7.96(2 \mathrm{H}, \mathrm{m}), 8.13(1 \mathrm{H}, \mathrm{s}), 8.19(2 \mathrm{H}, \mathrm{m}) .{ }^{13} \mathrm{C}$ NMR $\left(100 \mathrm{MHz}, \mathrm{CDCl}_{3}\right): \delta 16.21,16.84,17.07,19.12,20.71,22.22,26.85,28.20,33.24,35.25$, $36.66,37.85,38.36,42.19,42.45,47.30,49.53,52.19,56.33,57.87,59.44,120.48,121.77$, $125.08,125.90,125.99,130.36,133.29,134.01,136.91,137.18,138.65,140.12,144.59,147.78$, 178.75, 196.75, 198.37, 198.72. Anal. Calcd for $\mathrm{C}_{41} \mathrm{H}_{45} \mathrm{~N}_{3} \mathrm{O}_{5}$ : C, 74.63; H, 6.87; N, 6.37\%. Found: C, 74.89; H, 7.06; N, 6.07\%.

\section{(1-(4-Fluorophenyl)-1H-1,2,3-triazol-4-yl)methyl-16-isopropyl-1,4a-dimethyl-} $\mathbf{1 , 2 , 3 , 4 , 4 a , 4 b , 5 , 6 a , 7 , 1 2 , 1 2 a , 1 3 , 1 4 , 1 4 a - p e n t a d e c a h y d r o - 2 H - ( 6 , 1 2 b - e t h e n o ) b e n z o [ b ] c h r y s e n e - ~}$ 7,12-dione-1-carboxylate (7e). The yield is $180 \mathrm{mg}(71 \%), \mathrm{mp} 78-80{ }^{\circ} \mathrm{C}$ (hexane); $\mathrm{IR} \mathrm{(KBr,} v$, $\left.\mathrm{cm}^{-1}\right)$ : 1678, $1722(\mathrm{C}=\mathrm{O}) ;{ }^{1} \mathrm{H} \mathrm{NMR}\left(400 \mathrm{MHz}, \mathrm{CDCl}_{3}\right): \delta 0.48(3 \mathrm{H}, \mathrm{d}, J 6.5 \mathrm{~Hz}), 0.52(3 \mathrm{H}, \mathrm{s})$, $0.84(3 \mathrm{H}, \mathrm{d}, J 6.5 \mathrm{~Hz}), 0.92(1 \mathrm{H}, \mathrm{m}), 1.13(3 \mathrm{H}, \mathrm{s}), 1.21-1.88(1 \mathrm{H}, \mathrm{m}), 2.85(1 \mathrm{H}, \mathrm{d}, J 8.5 \mathrm{~Hz}), 3.05$ (1H, br.s), 3.24 (1H, dd, J 3.0, $8.5 \mathrm{~Hz}), 5.04(1 \mathrm{H}, \mathrm{s}), 5.32(2 \mathrm{H}, \mathrm{q}, J 12.8,16.8 \mathrm{~Hz}), 7.29(2 \mathrm{H}, \mathrm{m})$, $7.61(2 \mathrm{H}, \mathrm{m}), 7.69(1 \mathrm{H}, \mathrm{m}), 7.80(3 \mathrm{H}, \mathrm{m}), 8.00(1 \mathrm{H}, \mathrm{s}) .{ }^{13} \mathrm{C} \mathrm{NMR}\left(100 \mathrm{MHz}, \mathrm{CDCl}_{3}\right): \delta 16.20$, 16.82 , 17.06, 19.11, 20.71, 22.17, 28.18, 33.24, 35.22, 36.61, 37.81, 38.34, 42.21, 42.42, 47.26, $49.53,52.19,56.27,57.92,59.41,116.88,117.11,122.16,122.95,123.03,125.06,125.89$, $125.97,133.30,134.02,136.89,138.63,144.25,147.72,178.76,198.41,198.79$. Anal. Calcd for $\mathrm{C}_{39} \mathrm{H}_{42} \mathrm{FN}_{3} \mathrm{O}_{4}$ : C, 73.68; H, 6.66; F, 2.99, N, 6.61\%. Found: C, 73.87; H, 7.02; F, 3.08, N, 6.60\%.

\section{Pharmacological experiments}

The experiments were carried out using outbred female mice in accordance with the Guidelines 
for the Care and Use of Laboratory Animals. The animals were given standard granulated food and water ad libitum.

\section{Histamine-induced edema model}

Inflammatory edema was induced by subplanar injection of $0.05 \mathrm{ml} 0.01 \%$ histamine in water solution into the hind paw of female mice. The test compounds were administered intraperitoneally in $20 \mathrm{mg} / \mathrm{kg}$ b.w. dose (as aqueous-Tween- 80 suspension) one hour before the histamine injection. The reference agent indomethacin ('Fluka BioChemica') was administered in the $20 \mathrm{mg} / \mathrm{kg}$ dose. The control group of animals received a water-Tween- 80 mixture. The animals were sacrificed by cervical dislocation 5 hours after the phlogogenic agent injection, the mouse paws were cut off at the ankle joint and weighed. The ratio of the difference in weight between the treated and untreated hind paws to the weight of the untreated hind paw was used as an index of inflammation. The results were analyzed using "STATISTICA 6" software. The differences were significant at $p<0.05$. The anti-inflammatory activity was presented as a difference between $100 \%$ and percentage of inflammation index relative to control group.

\section{Acknowledgements}

This work was supported by the Interdisciplinary grant №51 of SB of the Russian Academy of Sciences (2012-2014), grant RFBR № 13-03-00129a, grant the Ministry of Education and Science of the Russian Federation (2014-2016) and the Chemical Service Centre of SB RAS. Work at FSU is supported by NSF, grant CHE-1152491.

\section{References}

1. Tolstikov, G. A.; Tolstikova, T. G.; Shults, E. E.; Tolstikov, S. E.; Khvostov, M. V. In Resin acids from Russian forest conifers. Chemistry and Pharmacology; Trofimov, B. A.; Ed.; Academic Publishing House “GEO”: Novosibirsk, 2011, 394pp (Russ.).

2. Kersten, P. J.; Kopper, B. J.; Raffa, K. F.; Illman, B. L. J. Chem. Ecol. 2006, 32, 2679. http://dx.doi.org/10.1007/s10886-006-9191-z

3. Trapp, S.; Croteau, R. Annu. Rev. Plant Physiol. Plant Mol. Biol. 2001, 52, 689. http://dx.doi.org/10.1146/annurev.arplant.52.1.689

4. Takahashi, N.; Kawada, T.; Goto, T.; Kim, Chu-S.; Taimatsu, A.; Egawa, K,; Yamamoto, T.; Jisaka, M.; Nishimura, K.; Yokota, K.; Yu, R.; Fushiki, T. FEBS Lett. 2003, 550, 190. http://dx.doi.org/10.1016/S0014-5793(03)00859-7

5. Kang, M. S.; Hirai, S.; Goto, T.; Kuroyanagi, K.; Lee, J. Y.; Uemura, T.; Ezaki, Y.; Takahashi, N.; Kawada, T. Biochem. Biophys. Res. Commun. 2008, 369, 333. 
6. Abdulla M. M. Monatsh. Chem. 2008, 139, 697.

http://dx.doi.org/10.1007/s00706-007-0804-1

7. Flekhter, O. B.; Tretyakova, E. V.; Makara, N.S.; Gabdrakhmanova, S.F.; Baschenko, N.Zh.; Galin, F. Z.; Zarudii, F. S.; Tolstikov, G. A. Pharm. Chem. J. 2003, 37, 142. http://dx.doi.org/10.1023/A:1024538715025

8. Kazakova, O. B.; Tretyakova, E. V.; Smirnova, I. E.; Spirikhin, I. V.; Tolstikov, G. A.; Chudov, I. V.; Bazekin, G. V.; Ismagilova, A. K. Russ. J. Bioorg. Chem. 2010, 36, 257. http://dx.doi.org/10.1134/S1068162010020160

9. Shults, E. E.; Raldugin, V. A.; Volcho, K. P.; Salakhutdinov, N. F.; Tolstikov, G. A. Russ. Chem. Rev. 2007, 76, 655. http://dx.doi.org/10.1070/RC2007v076n07ABEH003717

10. Vasilevsky, S. F.; Govdi, A. I.; Shult'ts, E. E.; Shakirov, M. M.; Alabugin I. V.; Tolstikov, G. A. Proc. Russ. Acad. Sci. 2009, 424, 631.

11. Dembitsky, V. M.; Levitsky, D. O. Nat. Prod. Commun. 2006, 405.

12. Galm, U.; Hager, M. H.; Van Lanen, S. G., Ju, J.; Thorson, J. S.; Shen, B. Chem. Rev. 2005, 105,739 .

http://dx.doi.org/10.1021/cr030117g

13. Breiner, B.; Kaya, K.; Roy, S.; Yang, W.-Y.; Alabugin, I. V. Org. Biomol. Chem. 2012, 10, 3974.

http://dx.doi.org/10.1039/c2ob00052k

14. Vasilevsky, S. F.; Govdi, A. I.; Shults, E. E.; Shakirov, M. M.; Sorokina, I. V.; Tolstikova, T. G.; Baev, D .S.; Tolstikov, G. A.; Alabugin, I. V. Bioorg. Med. Chem. 2009, 17, 516. http://dx.doi.org/10.1016/j.bmc.2009.05.059

15. Vasilevsky, S. F.; Govdi, A. I.; Sorokina, I. V.; Tolstikova, T. G.; Tolstikov, G. A.; Mamatuyk, V. I.; Alabugin, I. V. Bioorg. Med. Chem. Lett. 2011, 21, 62.

http://dx.doi.org/10.1016/j.bmcl.2010.11.072

16. Vafina, G. F.; Fazlyev, R.R. Galin, F.Z.; Spirikhin, L.V. Russ.J. Org. Chem. 2009, 45, 500. http://dx.doi.org/10.1134/S1070428009040058

17. Kazakova, O. B.; Tret'yakova, E. V.; Kukovinets, O. S.; Tolstikov, G. A.; Nazyrov, T.I.; Chudov, I.V.; Ismagilova, A. F. Russ. J. Bioorg. Chem. 2010, 36, 762.

http://dx.doi.org/10.1134/S1068162010060130

18. Kolb, H. C.; Finn, M. G.; Sharpless, B. K. Angew. Chem. Int. Ed. 2001, 40, 2004. http://dx.doi.org/10.1002/1521-3773(20010601)40:11<2004::AID-ANIE2004>3.0.CO;2-5

19. Finn, M. G.; Fokin, V. V. Chem. Soc. Rev. 2010, 39, 1221. http://dx.doi.org/10.1039/c003740k

20. Sletten, E. M.; Bertozzi, C. R. Angew. Chem. Int. Ed. 2009, 48, 6974. http://dx.doi.org/10.1002/anie.200900942

21. Debets, M. F.; van Berkel, S. S.; Dommerholt, J.; Dirks, A. J.; Rutjes, F. P. J. T.; van Delft, F.L. Acc. Chem. Res. 2011, 44, 805. http://dx.doi.org/10.1021/ar200059z 
22. Best, M. D.; Rowland, M. M.; Bostic, H. E. Acc. Chem. Res. 2011, 44, 686. http://dx.doi.org/10.1021/ar200060y

23. Codelli, J. A.; Baskin, J. M.; Agard, N. J.; Bertozzi, C. R. J. Am. Chem. Soc. 2008, 130, 11486.

http://dx.doi.org/10.1021/ja803086r

24. Ning, X.; Guo, J.; Wolfert, M. A. Boons, G.-J. Angew. Chem. Int. Ed. 2008, 47, 2253. http://dx.doi.org/10.1002/anie.200705456

25. Poloukhtine, A. A.; Mbua, N. E.; Wolfert, M. A.; Boons, G.-J.; Popik, V. V. J. Am. Chem. Soc. 2009, 131, 15769.

http://dx.doi.org/10.1021/ja9054096

26. Gold, B.; Schevchenko, N.; Bonus, N.; Dudley, G. B.; Alabugin, I. V. J. Org. Chem. 2012, $77,75$. http://dx.doi.org/10.1021/jo201434w

27. Gold, B.; Dudley, G. B.; Alabugin, I. V. J. Am. Chem. Soc. 2013, 135, 1558. http://dx.doi.org/10.1021/ja3114196

28. Porto de Oliveira Assis, Sh.; Targino da Silva, M.; de Oliveira, R. N.; deMenezes Lima, V. L. The ScientificWorld Journal 2012, Article ID 925925, 7 pages.

29. Csekei, M.; Novak, Z.; Kotschy, A. Tetrahedron 2008, 64, 975. http://dx.doi.org/10.1016/j.tet.2007.10.031

30. Endo, Y.; Songkram, C.; Yamasaki, R.; Tanatani, A.; Kagechika, H.; Takaishi, K.; Yamaguchi, K. J. Organomet. Chem. 2002, 657, 48.

http://dx.doi.org/10.1016/S0022-328X(02)01322-0

31. Lin, C.-H.; Wang, Y.-J.; Lee, C.-F. Eur. J. Org. Chem. 2010, 4368.

32. Takahashi, S.; Kuroyama, Y.; Sonogashira, K.; Hagihara, N. Synthesis 1980, 8, 627. http://dx.doi.org/10.1055/s-1980-29145

33. Ugi, I.; Perlinger, H.; Behringer, L. Chem. Ber. 1958, 55, 2330. http://dx.doi.org/10.1002/cber.19580911111

34. Pokhodylo, N. T.; Savka, R. D.; Matiichuk, V. S.; Obushak, N. D. Russ. J. Gen. Chem. 2009, $79,309$.

http://dx.doi.org/10.1134/S1070363209020248

35. Gilyarov, V. A.; Kabachnik, M. I. Zh. Obsh. Khim. 1966, 36, 282.

36. Henkel, K.; Weygand, F. Ber. 1943, 76, 812. http://dx.doi.org/10.1002/cber.19430760809

37. Moulin, Fr. Helv. Chim. Acta 1952, 5, 167. http://dx.doi.org/10.1002/hlca.19520350124 